\title{
Potential SARS-CoV-2 Nonstructural Protein 15 (NSP15) Inhibitors: Repurposing FDA-Approved Drugs
}

\section{Jason Y Tang ${ }^{1}$, Igor F. Tsigelny ${ }^{2-4 *}$, Jerry P. Greenberg ${ }^{2}$, Mark A. Miller², Valentina L. Kouznetsova ${ }^{2,4}$}

${ }^{1}$ REHS program, San Diego Supercomputer Center, UC San Diego, La Jolla, California, USA

${ }^{2}$ San Diego Supercomputer Center, UC San Diego, La Jolla, California., USA

${ }^{3}$ Department of Neurosciences, UC San Diego, La Jolla, California, USA

${ }^{4}$ BiAna. San Diego, California, USA

*Corresponding author: UC San Diego, 9500 Gilman Drive, La Jolla, CA 92093-0505, USA

Email: itsigeln@ucsd.edu

(Orcid ID: 0000-0002-7155-8947)

Running Head: NSP-15 inhibitors as COVID-19 Drugs

Word Count: 3743 


\begin{abstract}
Purpose: Severe acute respiratory syndrome coronavirus 2 (SARS-CoV-2) has caused millions of deaths worldwide, pushing the urgent need for an efficient treatment. Nonstructural protein 15 (NSP15) is a promising target due to its importance for SARS-CoV-2's evasion of the host's innate immune response.
\end{abstract}

Methods: Using the crystal structure of SARS-CoV-2 NSP15 endoribonuclease, we developed a pharmacophore model of the functional centers in the NSP15 inhibitor's binding pocket. With this model, we conducted data mining of the conformational database of FDA-approved drugs. The conformations of these compounds underwent 3D fingerprint similarity clustering, and possible conformers were docked to the NSP15 binding pocket. We also simulated docking of random compounds to the NSP15 binding pocket for comparison.

Results: This search identified 170 compounds as potential inhibitors of SARS-CoV-2 NSP15. The mean free energy of docking for the group of potential inhibitors were significantly lower than for the group of random compounds. Twenty-one of the compounds identified as potential NSP15 inhibitors were antiviral compounds used in the inhibition of a range of viruses, including MERS, SARS-CoV, and even SARS-CoV-2. Eight of the selected antiviral compounds in cluster A are pyrimidine analogues, six of which are currently used in a clinical setting. Four tyrosine kinase inhibitors were identified with potential SARS-CoV-2 inhibition, which is consistent with previous studies showing some kinase inhibitors acting as antiviral drugs.

Conclusions: We recommended testing of these 21 selected antiviral compounds for the treatment of COVID-19. 


\section{KEYWORDS}

SARS-CoV-2, Nonstructural Protein 15, NSP15, FDA-approved Drugs, Pharmacophore, Drug Repurposing, RNA uridylate-specific endoribonuclease, EndoU 


\section{INTRODUCTION}

Coronavirus disease-2019 (COVID-19) is a respiratory disease caused by SARS-CoV-2. As of August 1st, 2021, SARS-CoV-2 has cumulatively infected over 198 million people and killed over 4 million individuals in almost 200 countries and regions (https://coronavirus.jhu.edu). The serious threats to global public health and the economy presented by SARS-CoV-2 create an urgent need to identify novel tools to provide new pharmacologic leads that can improve survival for those already infected.

SARS-CoV-2 is a positive-sense, single-stranded, RNA betacoronavirus with a genome size of approximately 30kb. The genomic RNA contains a 5'-cap structure and a 3'-poly(A) tail. During infection, the genome is translated to generate viral polyproteins and transcribed to generate negative-sense RNA and subgenomic RNAs. The SARS-CoV-2 genome contains 14 open reading frames (ORFs) that encode 29 proteins, including nonstructural proteins (NSPs), structural proteins, and accessory proteins. The two main units, ORF1a and ORF1b are located at 5'-terminus and produce 16 NSPs through proteolytic cleavage by two viral proteases: the 3Clike protease $\left(3 \mathrm{CL}^{\mathrm{pro}}\right)$ and the papain-like protease $\left(\mathrm{PL}^{\mathrm{pro}}\right)$. NSPs are essential for RNA transcription, replication, translation, and suppressing the host antiviral response $\mathrm{e}^{1-3}$.

Targeting viral proteins to disrupt replication is an important approach in developing a therapeutic treatment against SARS-CoV-2 infection. Ideally, one can target highly conserved viral proteins that are unlikely to acquire resistance as the outbreak progresses. Recent studies report SARS-CoV-2 genomic variations in over $10 \%$ of isolated sequences, with the most frequent mutations being P323L in NSP12 and D641G in the spike protein ${ }^{4,5}$. In contrast, NSP15, an RNA uridylate-specific endoribonuclease (with a C-terminal region homologous to EndoU enzymes), is highly conserved, making it an attractive target for drug development. NSP15-like 
endoribonucleases are found in all coronavirus family members, suggesting its endonuclease function is critical for the viral life cycle. The amino-acid sequence alignment of NSP15 from SARS-CoV and SARS-CoV-2 showed $88 \%$ sequence identity and 95\% sequence similarity ${ }^{6}$. NSP15 recognizes uracil and cleaves single stranded RNA through an $\mathrm{Mn}^{2+}$ requiring transesterification reaction ${ }^{7}$. Recent studies indicate that NSP15 is not required for viral RNA synthesis; rather, NSP15 suppresses the host protective immune response through evasion of host dsRNA sensors ${ }^{8}$. Most recently, NSP15 was reported to participate in viral RNA processing by degrading viral polyuridine sequences. This may prevent the host immune sensing system from detecting viral RNA via cell pathogen-recognition receptors, which subsequently inhibits both direct and indirect antiviral effects ${ }^{9}$. These mechanisms are important for normal coronavirus infection of host cells. In the absence of NSP15 activity, viral replication is slowed significantly, and therefore NSP15 remains an attractive target for addressing SARS-CoV-2 infection ${ }^{10}$.

NSP15 is only active as a hexamer, which is formed as a dimer of trimers. The NSP15 monomer contains three domains: a N-terminal domain responsible for oligomerization, a middle domain, and a C-terminal domain, which contains the catalytic domain ${ }^{11}$. Binding sites of each of the catalytic domains are accessible despite hexamerization. A recent publication showed the first two crystal structures of SARS-CoV-2 NSP15 with $1.90 \AA$ and $2.20 \AA$ resolution $^{6}$. In the Cterminal catalytic domain of SARS-CoV-2 NSP15, the active site carries six key residues: His235, His250, Lys290, Thr341, Tyr343, and Ser294. Among of these residues, His235, His250, and Lys290 are suggested to constitute the catalytic triad for its nuclease activity. His250 acts as a general base to activate the 2'-OH of the ribose while His235 functions as a general acid to donate a proton to the leaving 5'-OH the ribose ${ }^{6,11}$. Ser294 together with Tyr343 determine uridine specificity. Ser294 is a key residue to recognize uracil and is assumed to interact with the 
carbonyl oxygen atom $\mathrm{O}_{2}$ of uracil, while Tyr343 orients the ribose of uridine for cleavage by van-der-Waals interactions ${ }^{11}$. In the crystal structure of the NSP15 citrate-bound form, the citrate ion forms hydrogen bonds with active site residues including His235, His250, Lys290, and Thr341 ${ }^{6}$. In the crystal structure of NSP15 complexed with uridine-5'-monophosphate (5'-UMP), 5'-UMP was found to interact with all six active site residues. The uridine base of 5'-UMP interacts with Tyr343 through van der Waals and forms hydrogen bonds with the nitrogen atom of Ser294, Lys290, and His250 ${ }^{12}$. This structural information is important for exploring binding of uridine analogues as a potential SARS-CoV-2 NSP15 inhibitors.

Tipiracil, an uracil derivative, is a thymidine phosphorylase inhibitor. It is an FDA-approved drug used with trifluridine to treat metastatic colorectal and gastric cancer. Previously, tipiracil has been reported to form hydrogen bonds with SARS-CoV-2 NSP15 active site residues Ser 294, Lys345, and His250 ${ }^{12}$. Tipiracil suppresses RNA nuclease activity of NSP15 and modestly inhibits SARS-CoV-2 virus replication in vitro without affecting viability of host cells most likely through competitive inhibition ${ }^{12}$. Moreover, recent in-silico-based approaches have identified other potential NSP15 inhibitors that await further structural and biochemical validation $^{13,14}$. The current COVID-19 pandemic brought attention to the repurposing of existing drugs and the rapid identification of candidate compounds. In this study, we use structure-based pharmacophore model and molecular docking to identify potential inhibitors of NSP15 by screening FDA approved drug database.

\section{MATERIALS AND METHODS}

The crystal structure of SARS-CoV-2 NSP15 endoribonuclease (PDB ID: 6WXC) in complex with the ligand tipiracil (5-chloro-6-(1-(2-iminopyrrolidinyl)methyl)uracil) was downloaded from the RCSB protein data bank. Using Molecular Operating Environment (MOE; CCG, Montreal, Canada), we analyzed the key binding site residues that are responsible for interaction 
between the NSP15 and tipiracil and employed a structure-based approach to construct our pharmacophore model of NSP15. The default forcefield is Amber 10: EHT with R Field solvation. Our pharmacophore model was created with seven features and excluded volume $\mathrm{R}=$ 1.6 A. It had 1 donor, 3 acceptors, 1 cationic atom\&donor, and 2 hydrophobic centroids. Based on this developed pharmacophore, we conducted a pharmacophore search on our conformational database of 2356 FDA approved drugs. Pharmacophore partial match was used for a 5 of 7 features search.

For multi-conformational docking of the selected compounds, we prepared the NSP15 structure with Protonate 3D application, isolated the ligand and pocket, visualized the space available for docked ligands, defined the binding pocket based on the known key residues for its nuclease activity and uridine specificity, and generated ligand conformations using the bond rotation method. The compounds were docked into the pocket using the Triangle Matcher Method and London dG scoring for placement; and the Induced Fit Method and GBVI/WSA dG scoring for refinement. Poses were ranked by GBVI/WSA binding free energy calculation in the S field. The 56 random control compounds were selected from the FDA drug database.

To further analyze ligand interactions for some of the above models, the structures were divided into ligand and protein pdb files. The separate structures were protonated: the protein with VMD (Visual Molecular Dynamics, v1.9.4) and the ligand with Avogadro v1.2.0. VMD was used to generate a psf (NAMD protein structure file) file for the protein and the Ligand Reader and Modeler from charmm-gui.org, was used to generate the psf and prm files for the ligand. VMD was then used with the CHARMM36 forcefield to re-combine the ligand and protein, thus solvating the structure and generating the required psf and pdb files ${ }^{15,16,17}$. NAMD v2.14 was used to run 100 steps of minimization followed by 100 ns of dynamics with 2fs/step (50,000,000 
iterations). The simulation conditions were rigid bonds involving hydrogen (rigidbonds set to "all"), a splitting distance of 12A between the short range and PME long range potential, Langevin dynamics at 310K with hydrogen atoms excluded (Langevin hydrogen set to "off"), and Periodic Boundary Conditions ${ }^{15,16,17}$.

\section{RESULTS}

\subsection{Pharmacophore model creation and search of drugs database}

A recent publication of the crystal structure of SARS-CoV-2 NSP15 endoribonuclease in complex with the ligand tipiracil provides detailed information regarding key residues responsible for the catalytic activity of NSP15 and its interactions with the potential ligands ${ }^{6}$. Based on the binding information for these key residues, we generated a pharmacophore model with potential functional centers that bind to the residues in the pocket (Figure 1A). 


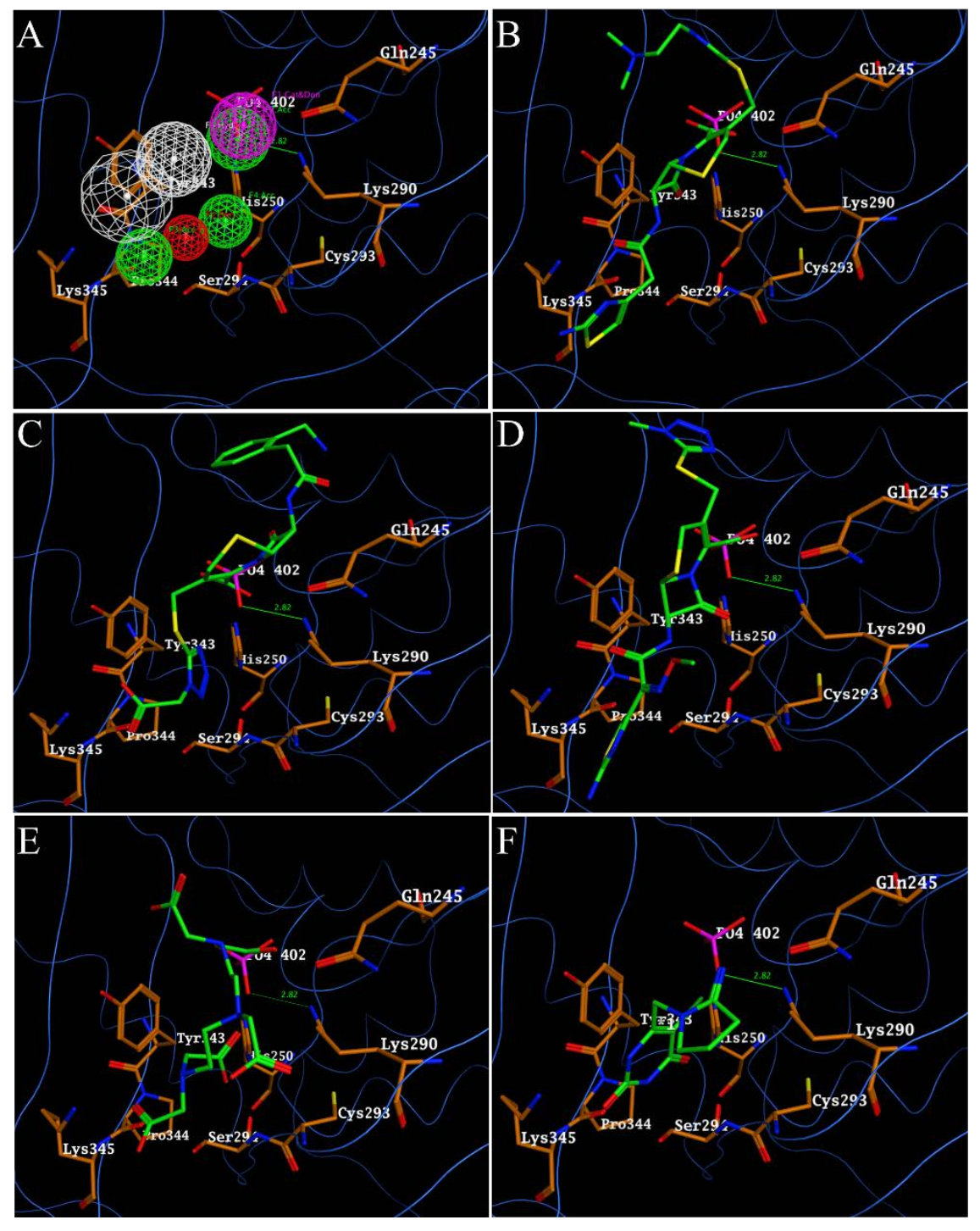

Figure 1. Pharmacophore of NSP15 binding pocket and binding poses of the best energy docked molecules. The model of pharmacophore (A) contains seven functional centers: one donor, three acceptors, one cationic atom\&donor, and two hydrophobic centroids. Binding poses of the drugs with the best scores. (B) Cefotiam, DFE $=-8.48 \mathrm{kcal} / \mathrm{mol}$. (C) Ceforanide, DFE $=-8.43$ $\mathrm{kcal} / \mathrm{mol}$. (D) Cefmenoxime, DFE $=-8.25 \mathrm{kcal} / \mathrm{mol}$. (E) Pentetic Acid, DFE $=-8.20 \mathrm{kcal} / \mathrm{mol}$. (F) Positive control, Tipiracil, DFE $=-5.14 \mathrm{kcal} / \mathrm{mol}$.

The pharmacophore search with a partial match 5 of 7 centers identified 803 compounds. We selected 170 compounds from the search based on the number of H-bonds and hydrophobic interactions in the best docking pose. Minimum three H-bonds and two hydrophobic interactions 
was the criteria for selection. Then we clustered the selected compounds using the Similarity Clustering of the MOE Database Viewer with a fingerprint GpiDAPH3 and similarity-overlap parameter $S O=45 \%$. The search identified three major hit clusters, containing ten or more compounds, along with several clusters containing less than ten compounds (from nine to two) and 36 single clusters with just one compound (Table 1). The two largest clusters (A and B) contain 16 and 35 compounds respectively, clusters C, D, E, F, G and H contain 11, 9, 7, 7, 5, and 5 compounds respectively; clusters I, J and K contain 4 compounds each, clusters L to V contain 2 to 3 compounds each, and there are 36 not clustered single compounds (Table 1). Flexible alignment of clusters was used to illustrate compounds common features (Figures 1 and 2). Cluster A mainly contains pyrimidine analogues that are known viral inhibitors. Cluster B mainly contains cephalosporin antibiotics. Cluster C mainly contains diuretic medications. Cluster D mainly contains ACE inhibitors and carbapenem antibiotics. Cluster E mainly contains beta blockers. Cluster F mainly contains nonsteroidal anti-inflammatory drugs. Cluster G mainly contains tyrosine kinase inhibitors. Cluster H mainly contains fluoroquinolones. Cluster I mainly contain diuretics. Cluster J mainly contains saccharide like compounds. Cluster K mainly contains prostaglandin like compounds.

The pharmacophore search with a partial match 5 of 7 centers identified 803 compounds. We selected 170 compounds from the search based on the number of H-bonds and hydrophobic interactions in the best docking pose. Minimum three H-bonds and two hydrophobic interactions was the criteria for selection. Then we clustered the selected compounds using the Similarity Clustering of the MOE Database Viewer with a fingerprint GpiDAPH3 and similarity-overlap parameter $S O=45 \%$. The search identified three major hit clusters, containing ten or more compounds, along with several clusters containing less than ten compounds (from nine to two) and 36 single clusters with just one compound (Table 1). The two largest clusters (A and B) 
contain 16 and 35 compounds respectively, clusters C, D, E, F, G and H contain 11, 9, 7, 7, 5, and 5 compounds respectively; clusters I, J and K contain 4 compounds each, clusters L to V contain 2 to 3 compounds each, and there are 36 not clustered single compounds (Table 1). Flexible alignment of clusters was used to illustrate compounds common features (Figures 1 and 2). Cluster A mainly contains pyrimidine analogues that are known viral inhibitors. Cluster B mainly contains cephalosporin antibiotics. Cluster C mainly contains diuretic medications. Cluster D mainly contains ACE inhibitors and carbapenem antibiotics. Cluster E mainly contains beta blockers. Cluster F mainly contains nonsteroidal anti-inflammatory drugs. Cluster G mainly contains tyrosine kinase inhibitors. Cluster H mainly contains fluoroquinolones. Cluster I mainly contain diuretics. Cluster $\mathrm{J}$ mainly contains saccharide like compounds. Cluster $\mathrm{K}$ mainly contains prostaglandin like compounds. 
Table 1. Drug candidates clustered by fingerprint similarity.

\begin{tabular}{|c|c|c|c|c|c|c|}
\hline \multicolumn{6}{|c|}{ CLUSTERS } & \multirow{2}{*}{ Single Drug } \\
\hline A & B & $\mathrm{C}$ & $\mathbf{F}$ & $\mathbf{K}$ & $\mathrm{S}$ & \\
\hline Aminoglutethimide & \multirow{4}{*}{$\begin{array}{l}\text { Amoxicillin } \\
\text { Ampicillin } \\
\text { Azlocillin } \\
\text { Betiatide }\end{array}$} & \multirow{6}{*}{$\begin{array}{l}\text { Bendroflumethiazide } \\
\text { Benzthiazide } \\
\text { Chlorothiazide } \\
\text { Cyclopenthiazide } \\
\text { Furosemide } \\
\text { Hydrochlorothiazide }\end{array}$} & \multirow{7}{*}{$\begin{array}{l}\text { Azathioprine } \\
\text { Dantrolene } \\
\text { Lornoxicam } \\
\text { Meloxicam } \\
\text { Nitazoxanide } \\
\text { Nitrofurantoin } \\
\text { Tenoxicam }\end{array}$} & \multirow{4}{*}{$\begin{array}{l}\text { Alprostadil } \\
\text { Dinoprostone } \\
\text { Hydrocortamate } \\
\text { Losartan }\end{array}$} & Cimetidine & \multirow{7}{*}{$\begin{array}{l}\text { Almotriptan } \\
\text { Amlodipine } \\
\text { Amprenavir } \\
\text { Atorvastatin } \\
\text { Cabergoline } \\
\text { Capecitabine } \\
\text { Citric acid }\end{array}$} \\
\hline Biotin & & & & & Famotidine & \\
\hline Brivudine & & & & & & \\
\hline Butabarbital & & & & & $\mathbf{T}$ & \\
\hline Butalbital & Cefaclor & & & & \multirow{3}{*}{$\begin{array}{l}\text { Sulfadoxine } \\
\text { Sulfametopyrazine }\end{array}$} & \\
\hline Edoxudine & Cefadroxil & & & $\mathbf{L}$ & & \\
\hline Ethosuximide & Cefamandole & Methyclothiazide & & \multirow{4}{*}{$\begin{array}{l}\text { Labetalol } \\
\text { Pirbuterol } \\
\text { Salbutamol }\end{array}$} & & \\
\hline Floxuridine & $\begin{array}{l}\text { Cefamandole } \\
\text { nafate }\end{array}$ & Metolazone & & & $\mathbf{U}$ & Dexrazoxane \\
\hline Glutethimide & Cefapirin & Polythiazide & $\mathbf{G}$ & & Dexpanthenol & Dofetilide \\
\hline Propylthiouracil & Cefazolin & Quinethazone & \multirow{6}{*}{$\begin{array}{l}\text { Afatinib } \\
\text { Dacomitinib } \\
\text { Dasatinib } \\
\text { Gefitinib } \\
\text { Lapatinib }\end{array}$} & & \multirow[t]{2}{*}{ Pantothenic acid } & Doravirine \\
\hline Stavudine & Cefdinir & Trichlormethiazide & & $\mathbf{M}$ & & Enoximone \\
\hline \multirow{24}{*}{$\begin{array}{l}\text { Telbivudine } \\
\text { Tipiracil } \\
\text { Trifluridine } \\
\text { Uracil mustard } \\
\text { Zidovudine }\end{array}$} & Cefditoren & & & Bicisate & $\mathbf{V}$ & Entacapone \\
\hline & Cefmenoxime & D & & Edetic Acid & \multirow{23}{*}{$\begin{array}{l}\text { Peramivir } \\
\text { Pinacidil }\end{array}$} & Eprosartan \\
\hline & Cefmetazole & Doripenem & & Pentetic Acid & & Famciclovir \\
\hline & Cefonicid & \multirow{9}{*}{$\begin{array}{l}\text { Ertapenem } \\
\text { Etacrynic acid } \\
\text { Imipenem } \\
\text { Lisdexamfetamine } \\
\text { Meropenem } \\
\text { Perindopril } \\
\text { Ramipril } \\
\text { Spirapril }\end{array}$} & & & & Fursultiamine \\
\hline & Ceforanide & & $\mathbf{H}$ & $\mathbf{N}$ & & Indacaterol \\
\hline & Cefotaxime & & \multirow{6}{*}{$\begin{array}{l}\text { Gatifloxacin } \\
\text { Levofloxacin } \\
\text { Nalidixic acid } \\
\text { Nedocromil } \\
\text { Trovafloxacin }\end{array}$} & Riboflavin & & L-Citrulline \\
\hline & Cefotiam & & & Sapropterin & & Macitentan \\
\hline & Cefoxitin & & & & & Mannitol \\
\hline & Cefpirome & & & $\mathbf{O}$ & & Methocarbamol \\
\hline & Cefpodoxime & & & Carglumic acid & & Milrinone \\
\hline & Cefprozil & & & Glutathione & & Minocycline \\
\hline & \multirow{4}{*}{$\begin{array}{l}\text { Ceftibuten } \\
\text { Ceftizoxime } \\
\text { Cephaloglycin } \\
\text { Cephalothin }\end{array}$} & & I & & & Nialamide \\
\hline & & $\mathbf{E}$ & \multirow{5}{*}{$\begin{array}{l}\text { Gliclazide } \\
\text { Tolazamide } \\
\text { Tolbutamide } \\
\text { Torasemide }\end{array}$} & $\mathbf{P}$ & & Oseltamivir \\
\hline & & \multirow{11}{*}{$\begin{array}{l}\text { Acebutolol } \\
\text { Atenolol } \\
\text { Bisoprolol } \\
\text { Celiprolol } \\
\text { Metipranolol } \\
\text { Nadolol } \\
\text { Timolol }\end{array}$} & & Glimepiride & & Oxeladin \\
\hline & & & & Glyburide & & Ranolazine \\
\hline & Cloxacillin & & & & & Ritiometan \\
\hline & Cyclacillin & & & $\mathbf{Q}$ & & Rutin \\
\hline & Dicloxacillin & & $\mathbf{J}$ & Amikacin & & Sofosbuvir \\
\hline & Flucloxacillin & & \multirow{6}{*}{$\begin{array}{l}\text { Acarbose } \\
\text { Gaxilose } \\
\text { Lactulose } \\
\text { Mannitol } \\
\text { busulfan }\end{array}$} & Tobramycin & & Spectinomycin \\
\hline & Loracarbef & & & & & Streptomycin \\
\hline & Methicillin & & & $\mathbf{R}$ & & Streptozocin \\
\hline & Mezlocillin & & & Acemetacin & & Sulpiride \\
\hline & $\begin{array}{l}\text { Nafcillin } \\
\text { Penicillin V }\end{array}$ & & & Tolmetin & & $\begin{array}{l}\text { Tazobactam } \\
\text { Uridine } \\
\text { triacetate }\end{array}$ \\
\hline & & & & & & Xanthinol \\
\hline
\end{tabular}

Note: Drugs benzonatate, cefotetan, and fosamprenavir were not stable in MD simulation and thus excluded. 

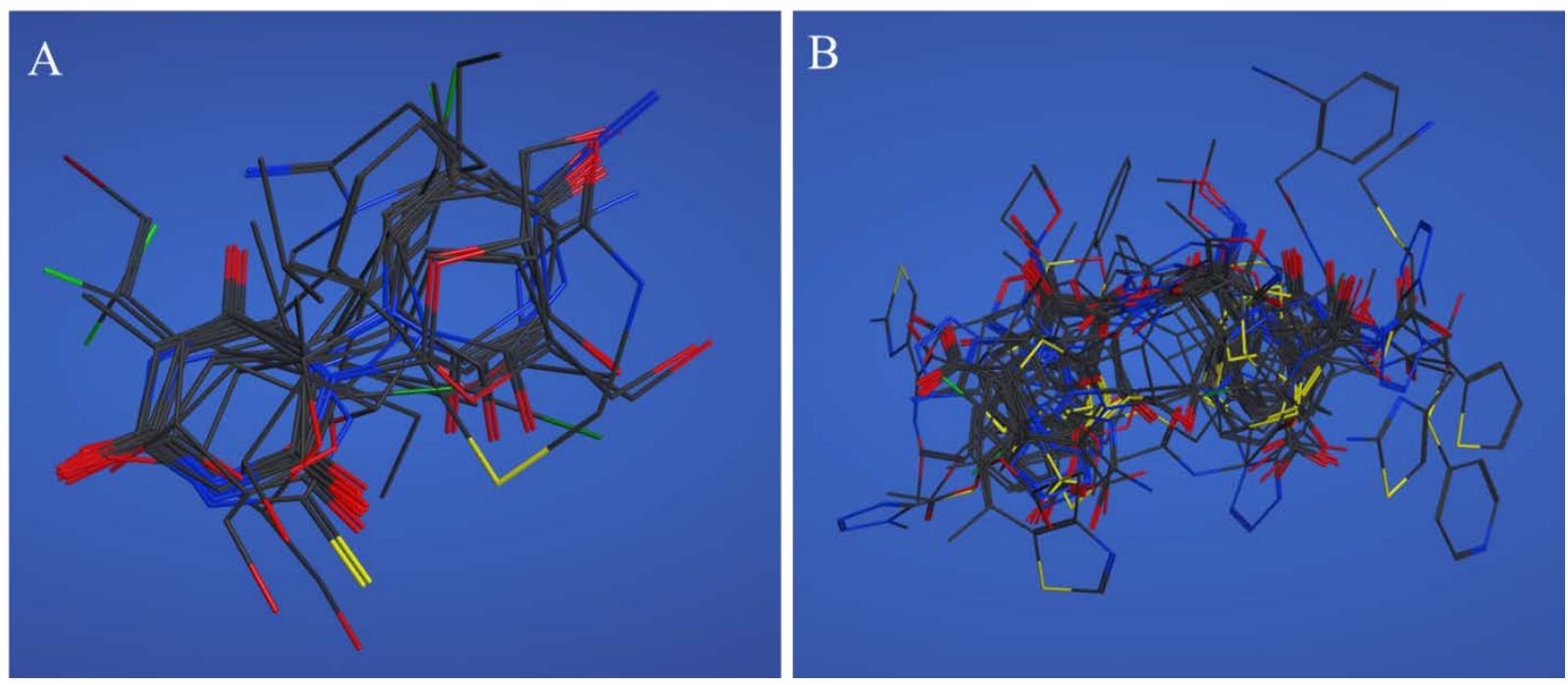

Figure 2. Flexible alignments of compounds in clusters selected by the pharmacophore-based search of possible drug candidates in the conformational database of FDA-approved drugs. (A) Cluster A (16 compounds). (B) Cluster B (35 compounds).

\subsection{Computational docking}

For docking the selected compounds, we used the crystal structure of SARS-CoV-2 NSP15 endoribonuclease (PDB ID: 6WXC), which was imported into MOE. After the structure preparation and the model's binding pocket was defined, based on known key residues for its nuclease activity and uridine specificity, ligand conformations were generated using the bond rotation method. These were then docked into the site with the Triangle Matcher method and ranked with the London dG scoring function. The retain option specifies the number of poses (30) to pass to the refinement, which is for energy minimization in the pocket, before rescoring with the Induced Fit method and GBVI/WSA dG scoring function. To validate docking, 56 random control compounds were selected from the FDA drug database, using a random number generator without repetitions.

The values of docking free energies of the selected and random compounds are shown in Figure

3. The means of the selected and random compounds are $-6.50 \mathrm{kcal} / \mathrm{mol}$ and $-5.79 \mathrm{kcal} / \mathrm{mol}$, 
respectively. Furthermore, the $p$ value of one tail for selected vs random compounds is $1.31 \mathrm{E}-06$. Energies of interaction with the NSP15 active site are shown in Table 2 and Table S1.

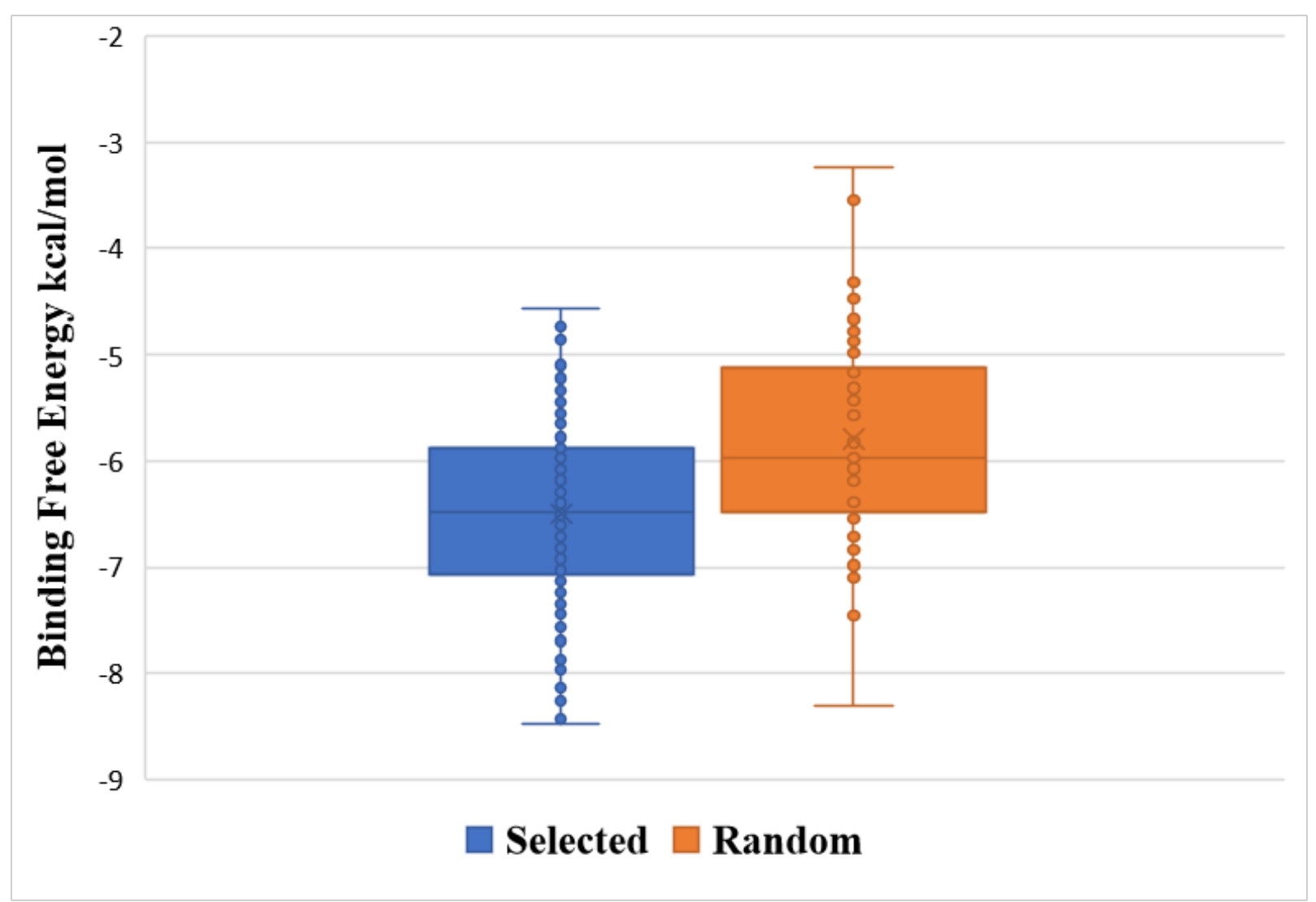

Figure 3. Free energies of docking interaction of selected and random compounds with SARSCoV-2 NSP15. The means of the selected and random compounds are -6.50 and $-5.79 \mathrm{kcal} / \mathrm{mol}$, respectively. The p value of one tail is $1.31 \mathrm{E}-06$. 
Table 2. List of selected compounds sorted by their energies of interaction with SARS-CoV-2 NSP15 in the docked positions. All compounds shown have an energy less than -7. DFE:

Docking free energy.

\begin{tabular}{|l|l|l|l|l|l|}
\hline Drug Name & $\begin{array}{l}\text { DFE } \\
\text { kcal/mol }\end{array}$ & Cluster & Drug Name & $\begin{array}{l}\text { DFE } \\
\text { kcal/mol }\end{array}$ & Cluster \\
\hline Cefotiam & -8.48 & B & Gliclazide & -7.33 & I \\
Ceforanide & -8.43 & B & Streptomycin & -7.31 & S \\
Cefmenoxime & -8.25 & B & Amprenavir & -7.29 & S \\
\hline Pentetic Acid & -8.20 & M & Minocycline & -7.28 & S \\
\hline Cefonicid & -8.14 & B & Azlocillin & -7.26 & B \\
\hline Cephaloglycin & -7.97 & B & Dasatinib & -7.26 & G \\
\hline Cefamandole nafate & -7.95 & B & Acarbose & -7.25 & J \\
\hline Cefamandole & -7.94 & B & Cefditoren & -7.24 & B \\
\hline Atorvastatin & -7.87 & S & Meropenem & -7.19 & D \\
\hline Cefazolin & -7.79 & B & Cefpirome & -7.18 & B \\
\hline Ertapenem & -7.70 & D & Macitentan & -7.13 & S \\
\hline Doripenem & -7.66 & D & Cefdinir & -7.12 & B \\
\hline Glyburide & -7.65 & P & Betiatide & -7.12 & B \\
\hline Cefmetazole & -7.62 & B & Alprostadil & -7.12 & K \\
\hline Dinoprostone & -7.56 & K & Cefoxitin & -7.11 & B \\
\hline Spirapril & -7.52 & D & Acemetacin & -7.11 & R \\
\hline Cefotaxime & -7.52 & B & Ramipril & -7.09 & D \\
\hline Cefapirin & -7.50 & B & Ranolazine & -7.07 & S \\
\hline Cephalothin & -7.48 & B & Afatinib & -7.06 & G \\
\hline Ceftibuten & -7.44 & B & Losartan & -7.06 & K \\
\hline Lapatinib & -7.42 & G & Cefadroxil & -7.03 & B \\
\hline Cefprozil & -7.39 & B & Methicillin & -7.03 & B \\
\hline Cefpodoxime & -7.37 & B & Sofosbuvir & -7.03 & S \\
\hline Mezlocillin & -7.34 & B & & & \\
\hline & & & & & \\
\hline
\end{tabular}

\subsection{Molecular dynamics simulations}

We selected the top three compounds in docking energies to further analyze stability of ligand interactions: cefmenoxime, cefotiam, and ceforanide. The final configuration of the compoundprotein complexes resulting in this MD simulations are shown in Figure 4 and Table 3. 
Cefmenoxime (Figure 4A and 4D) had 6 major ligand interactions with NSP15, the shortest distance of which was 2.73 with the residue Lys290. Cefotiam (Figure 4B and 4E) had 4 major ligand interactions with NSP15, the shortest distance of which was 2.693 with the residue Leu246. Finally, ceforanide (Figure 4C and 4F) had 2 major ligand interactions with NSP15, the shortest distance of which was 2.70 with the residue Lys290. Figure 5 shows the measures though MD distances between the NZ atom of LYZ290 of protein with the geometric center of these compounds. One can see that these distances pretty stable during MD simulations.
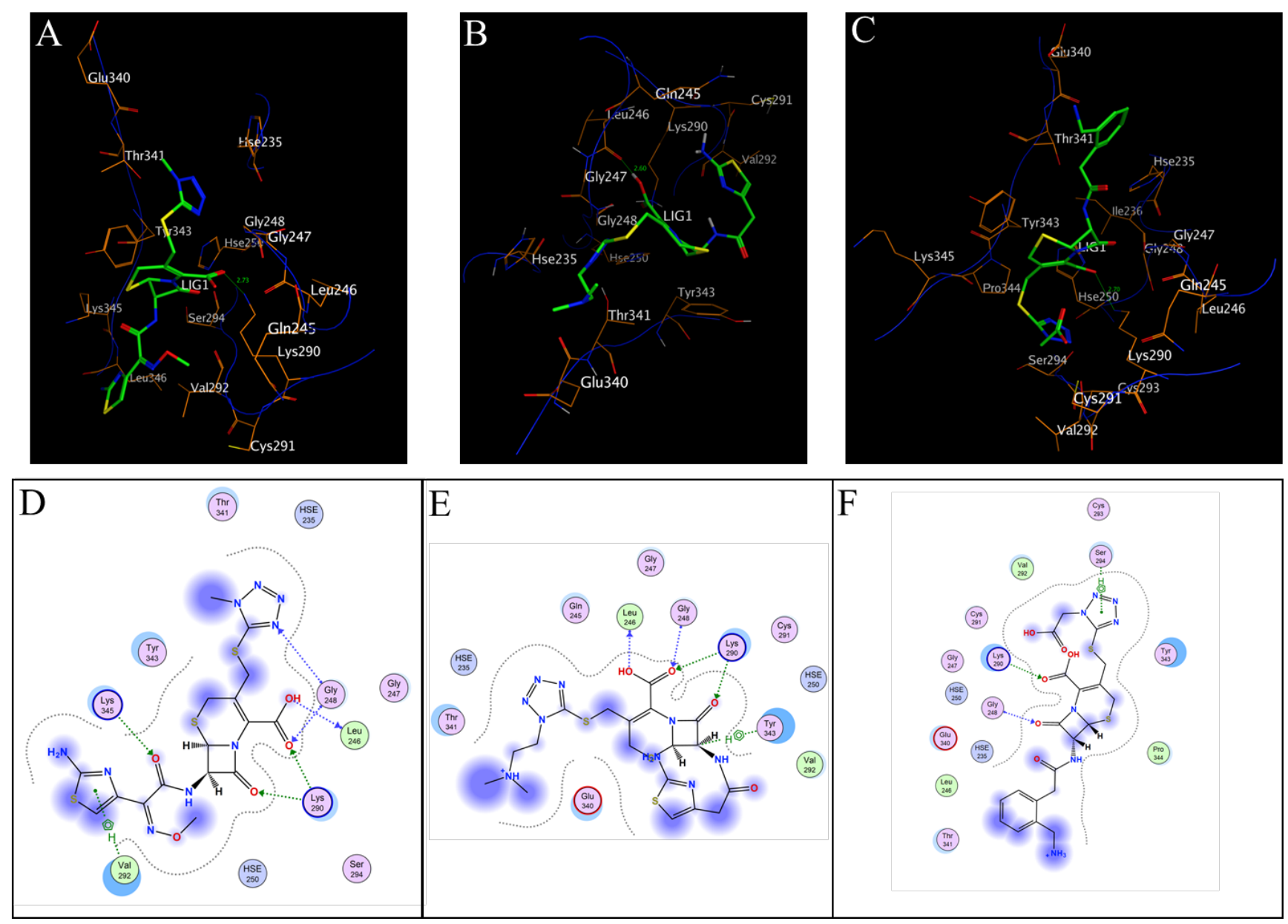

Figure 4. Further analysis of cefmenoxime (A and D), cefotiam (B and E), and ceforanide (C and F) through showing ligand interactions with the NSP15 binding pocket. 
Table 3. List of ligand interactions with NSP15 binding pocket measuring distance and energy for the compounds in Figure 4.

\begin{tabular}{|c|c|c|c|c|c|c|}
\hline \multicolumn{7}{|c|}{ Cefmenoxime } \\
\hline \multicolumn{2}{|c|}{ Ligand } & \multicolumn{2}{|c|}{ Receptor } & \multirow{2}{*}{$\begin{array}{c}\text { Interaction } \\
\text { H-donor }\end{array}$} & \multirow{2}{*}{$\begin{array}{c}\text { Distance } \\
2.83\end{array}$} & \multirow{2}{*}{$\frac{\mathrm{E}(\mathrm{kcal} / \mathrm{mol})}{-5.0}$} \\
\hline OXT & 6 & $\mathrm{O}$ & Leu246 & & & \\
\hline $\mathrm{O} 1$ & 7 & $\mathrm{~N}$ & Gly248 & H-acceptor & 2.79 & -0.5 \\
\hline $\mathrm{O} 1$ & 7 & NZ & Lys290 & H-acceptor & 2.73 & -6.7 \\
\hline $\mathrm{O} 2$ & 10 & NZ & Lys290 & H-acceptor & 2.80 & -4.0 \\
\hline O3 & 14 & CE & Lys345 & H-acceptor & 3.51 & -0.8 \\
\hline N9 & 33 & $\mathrm{~N}$ & Gly248 & H-acceptor & 3.46 & -0.6 \\
\hline \multicolumn{7}{|c|}{ Cefmenoxime } \\
\hline \multicolumn{2}{|c|}{ Ligand } & \multicolumn{2}{|c|}{ Receptor } & Interaction & Distance & $\mathrm{E}(\mathrm{kcal} / \mathrm{mol})$ \\
\hline OXT & 6 & $\mathrm{O}$ & Leu246 & H-donor & 2.69 & -2.8 \\
\hline $\mathrm{O} 1$ & 7 & $\mathrm{~N}$ & Gly248 & H-acceptor & 2.77 & -2.0 \\
\hline $\mathrm{O} 1$ & 7 & NZ & Lys290 & H-acceptor & 2.96 & -3.3 \\
\hline $\mathrm{O} 2$ & 10 & NZ & Lys290 & H-acceptor & 2.82 & -7.9 \\
\hline \multicolumn{7}{|c|}{ Ceforanide } \\
\hline \multicolumn{2}{|c|}{ Ligand } & \multicolumn{2}{|c|}{ Receptor } & Interaction & Distance & $\mathrm{E}(\mathrm{kcal} / \mathrm{mol})$ \\
\hline $\mathrm{O} 1$ & 6 & $\mathrm{NZ}$ & Lys290 & H-acceptor & 2.70 & -4.6 \\
\hline $\mathrm{O} 2$ & 10 & $\mathrm{~N}$ & Gly248 & H-acceptor & 2.71 & -5.8 \\
\hline
\end{tabular}

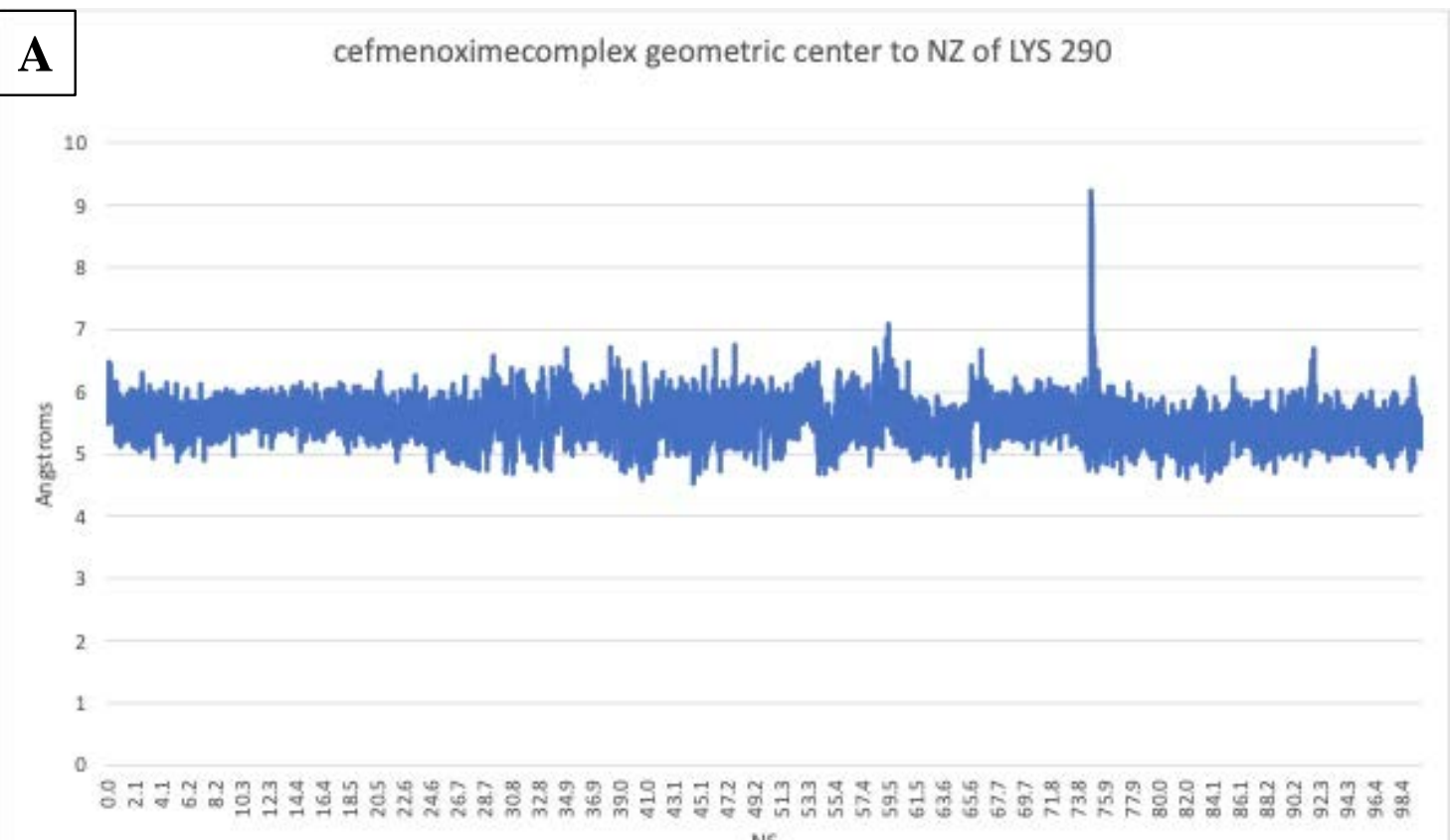


B cefotiamcomplex geometric center to NZ of LYS 290

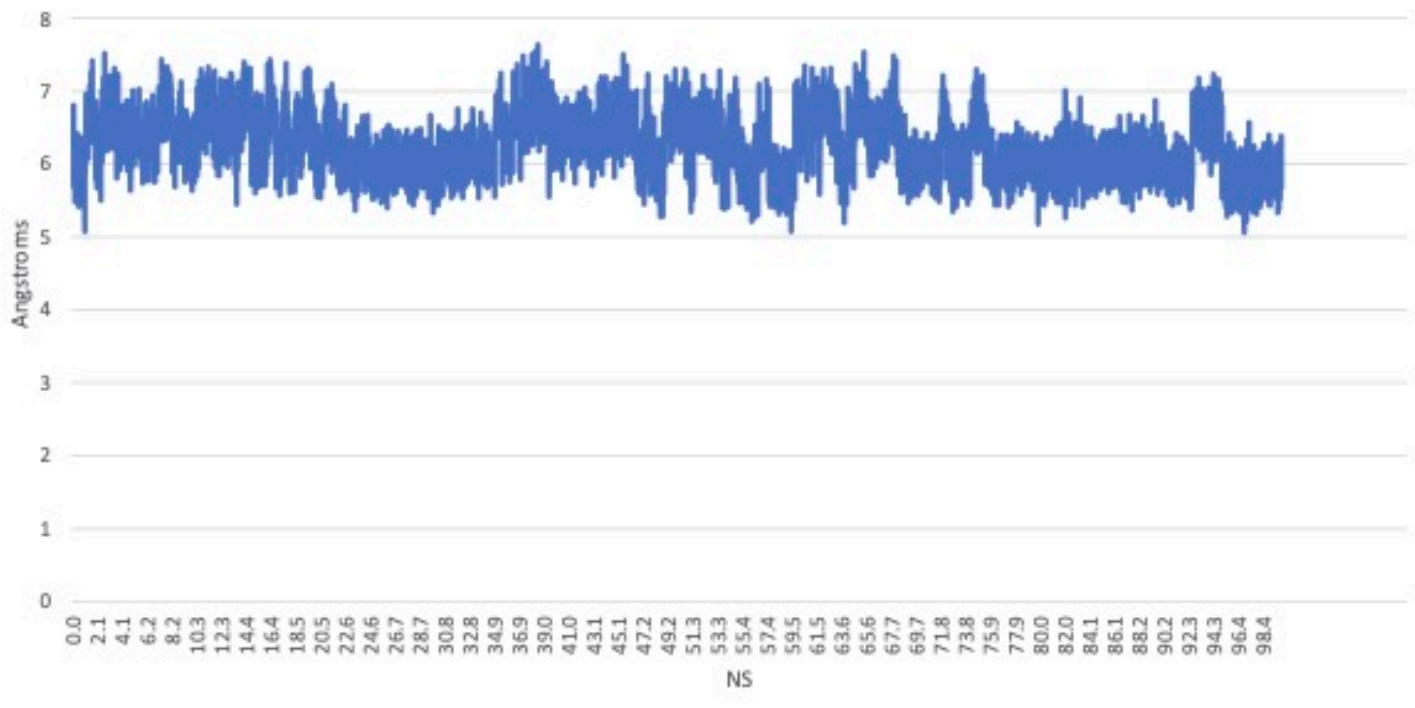

C ceforanidecomplex geometric center to NZ of LYS 290

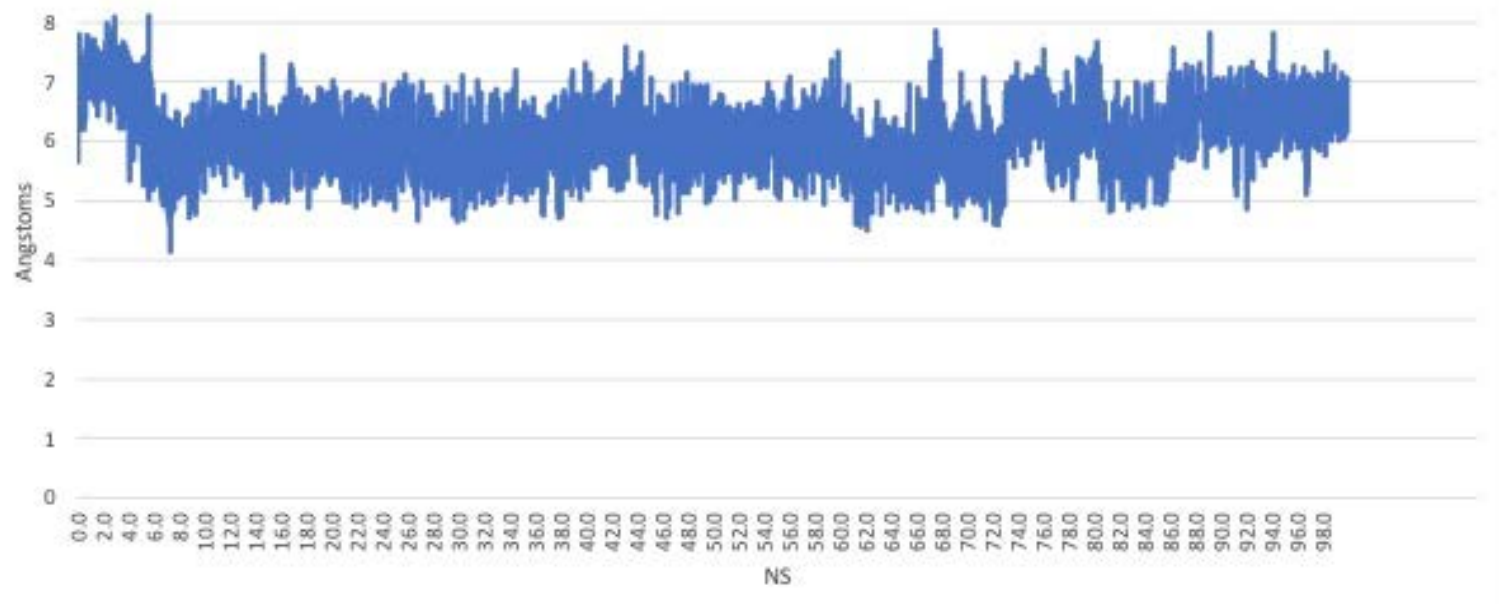

Figure 5. Distances during 100 ns MD between the atom NZ of the residue LYS290 of protein to the geometric centers of: (A) Cefmenoxime, (B) Cefotiam, (C) Ceforanide. The plots show stability of positions of these compounds. 


\section{DISCUSSION}

Based on the crystal structure of SARS-CoV-2 NSP15 endoribonuclease in complex with tipiracil, we have developed a pharmacophore model of NSP15's binding pocket, including key residues for its nuclease activity and uridine specificity. Using this model, we conducted a pharmacophore search of our conformational database of FDA-approved drugs. In the search, 170 compounds were selected, clustered, and were then used for flexible docking into the NSP15 active site pocket in catalytic domain. Twenty-one of the compounds identified as potential NSP15 inhibitors were antiviral compounds used against a range of viruses, including HIV, HCV, HBV, influenza, and HSV. Some of them also demonstrated inhibition activity for MERS, SARS-CoV, and SARS-CoV-2 (Table 4).

Table 4. List of selected compounds with known antiviral activity.

\begin{tabular}{|l|l|l|l|l|l|}
\hline \multicolumn{1}{|c|}{ Cluster A } & Cluster F & Cluster G & Cluster W & Cluster U & Single drugs \\
\hline Trifluridine & Nitazoxanide & Gefitinib & Peramivir & Amprenavir & Oseltamivir \\
Zidovudine & & Dasatinib & & & Famciclovir \\
Floxuridine & & Lapatinib & & & Sofosbuvir \\
Stavudine & & Afatinib & & & Doravirine \\
Telbivudine & & & & & \\
Brivudine & & & & & \\
Edoxudine & & & & & \\
Tipiracil & & & & & \\
\hline
\end{tabular}

According to the DrugVirus.info database ${ }^{18}, 13$ of the antiviral compounds selected by the pharmacophore-based search showed activity against a total of 40 viruses in cell-culture, animal, and clinic models (Figure 6). The other eight antiviral compounds were not in the database. A previous study did not identify any of these compounds as potential NSP15 inhibitors, and their top selected drugs did not show antiviral activity ${ }^{14}$. Differences in methodology may explain these discrepancies of results. Specifically, Chandra and co-authors used NSP15 PDB ID 6W01 structure with a citrate ion $^{14}$; we used crystal structure of NSP15 in complex with tipiracil that 
binds to NSP15 uracil site. We assume that the pharmacophore model generated on this protein structure includes the key features responsible for ligand interaction with residues in NSP15 active site. We did notice that tipiracil, the positive control, did not have a low free energy. However, an in-vitro study confirmed that tipiracil can inhibit uracil binding to the NSP15 active site presumably through competitive inhibition and modestly suppress SARS-CoV-2 viral replication in cellular assays ${ }^{12}$. Cluster A includes six pyrimidine analogues that are currently used as viral inhibitors: HIV reverse transcriptase inhibitors-zidovudine and stavudine, HBV DNA polymerase inhibitor-telbivudine, and HSV DNA polymerase inhibitors-brivudine, edoxudine, and trifluridine (Table 4 and Figure 6). The other two drugs in cluster A, tipiracil ${ }^{12}$ and floxuridine $\mathrm{e}^{19}$, are anticancer drugs that have antiviral properties. All these pyrimidine analogues are polymerase inhibitors, which is a major class of antiviral drugs.

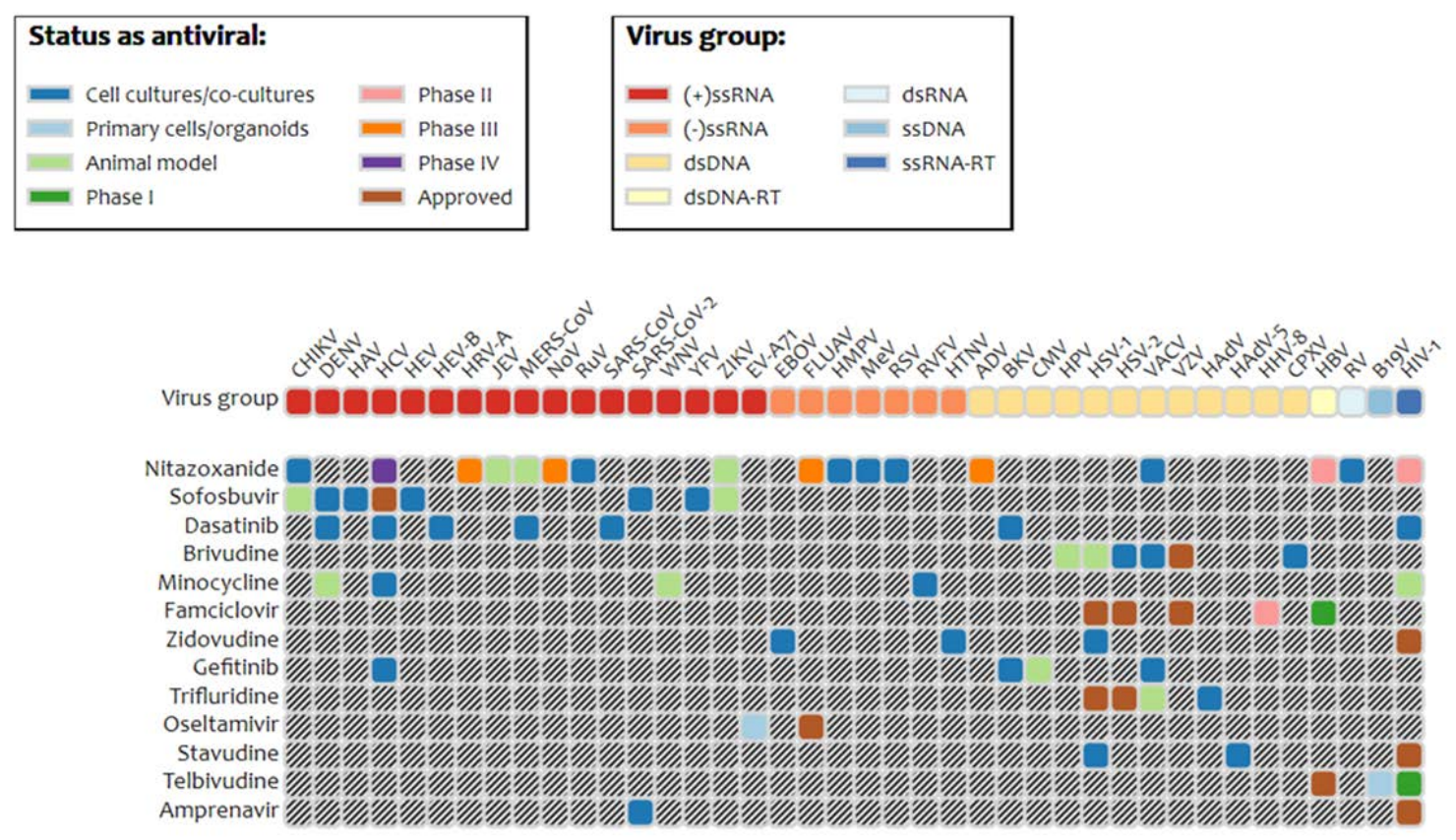

Figure 6. A chart representing 13 of the selected antiviral drugs and their use against different viruses. Obtained using DrugVirus.info database ${ }^{18}$. 
These results support using NSP15's pharmacophore features to identify potential antiviral compounds containing a pyrimidine-like scaffold and further development of nucleotide-like drugs with higher affinity for the active site of NSP15. Recent studies demonstrated that tyrosine kinase inhibitors have antiviral potential through inhibition of key kinases required for viral entry and reproduction ${ }^{20,21}$. Thus, repurposing receptor tyrosine kinase inhibitors is an effective strategy in the fight against COVID-1922. Our pharmacophore model successfully identified four tyrosine kinase inhibitors with antiviral activity in cluster G, the binding affinities of which are high. Dasatinib, an approved drug for chronic myelogenous leukemia (CML), has activity against both MERS-CoV and SARS-CoV in vitro and possible protection against SARS-CoV-2 infection ${ }^{23,24}$. EGFR inhibitor gefitinib has demonstrated in vitro activity against HCV, BKV, CMV, and VACV (Figure 6). Lapatinib was just recently found to potently inhibit SARS-CoV-2 replication at clinical doses, strongly supporting our screening result ${ }^{25}$.

Promising antiviral drugs from cluster $\mathrm{U}$ includes HIV proteinase inhibitor amprenavir. Specifically, amprenavir has a free energy of $-7.29 \mathrm{kcal} / \mathrm{mol}$ and modestly inhibits replication of SARS-CoV-2 in vitro ${ }^{26}$. Outside of clusters A, G, and U, other antiviral drugs include influenza neuraminidase inhibitors peramivir and oseltamivir, HIV non-nucleoside reverse transcriptase inhibitor doravirine, and HCV NS5B polymerase inhibitor sofosbuvir, which displayed activity against SARS-CoV-2 ${ }^{27}$.

Interesting to note that some of randomly selected FDA-approved drugs had free energies below $-7.00 \mathrm{kcal} / \mathrm{mol}$, namely gadoxetate $(-8.31 \mathrm{kcal} / \mathrm{mol})$, iohexol $(-7.45 \mathrm{kcal} / \mathrm{mol})$, and chlortetracycline $(-7.11 \mathrm{kcal} / \mathrm{mol})$ (Table S1). These compounds also can be potential inhibitors of NSP15. 


\section{CONCLUSION}

Given the severity of the COVID-19 pandemic, we need a fast way of finding treatment. Identification of FDA-approved drugs to inhibit SARS-CoV-2 could lead to advances in this field. Though this study is limited due to only using computer-based screening, the implications of the 170 compounds is a key step in finally finding a treatment. Twenty-one of these drugs have known antiviral properties, some of which have demonstrated inhibition of SARS-CoV-2 in vitro. We recommended testing of selected compounds for the treatment of COVID-19, especially those in clusters $A, G$, and $U$.

\section{ACKOWLEDGEMENTS}

We would like to thank the people of San Diego Supercomputer Center and CCG (Montreal, Canada).

\section{CONFLICT OF INTERESTS}

The authors declare that they have no competing interests.

\section{AUTHOR CONTRIBUTIONS}

JYT participated in design of the study, performed the docking computational experiments, analyzed the data, and drafted the manuscript. IFT and VLK proposed the study concept, conducted pharmacophore development, databases search, project guidance, and manuscript writing and editing. MAM participated in designing of the study concept, wrote and edit the manuscript. All authors reviewed and approved the final manuscript.

\section{ORCID}

Igor F. Tsigelny http://orcid.org/0000-0002-7155-8947 


\section{REFERENCES}

1. Romano M, Ruggiero A, Squeglia F, et al. A structural view of SARS-CoV-2 RNA replication machinery: RNA synthesis, proofreading and final capping. Cells. 2020; 9(5): 1267.

2. Kim D, Lee JY, Yang JS, et al. The architecture of SARS-CoV-2 transcriptome. Cell. 2020; 181(4): 914-921.

3. Yao H, Song Y, Chen Y, et al. Molecular architecture of the SARS-CoV-2 virus. Cell. 2020; 183(3): 730-738.

4. Ugurel OM, Ata O, Turgut-Balik D. An updated analysis of variations in SARS-CoV-2 genome. Turk J Biol. 2020; 44(3): 157-170.

5. Toyoshima $\mathrm{Y}$, Nemoto $\mathrm{K}$, Matsumoto $\mathrm{S}$, et al. SARS-CoV-2 genomic variations associated with mortality rate of COVID-19. J Hum Genet. 2020; 65: 1075-1082.

6. Kim Y, Jedrzejczak R, Maltseva NI, et al. Crystal structure of Nsp15 endoribonuclease NendoU from SARS-CoV-2. Protein Sci. 2020; 29(7): 1596-1605.

7. Bhardwaj K, Sun J, Holzenburg A, et al. RNA recognition and cleavage by the SARS coronavirus endoribonuclease. J Mol Biol. 2006; 361(2): 243-256.

8. Deng X, Hackbart M, Mettelman R, et al. Coronavirus nonstructural protein 15 mediates evasion of dsRNA sensors and limits apoptosis in macrophages. Proc Natl Acad Sci U S A. 2017; 114(21): E4251-E4260.

9. Hackbart M, Deng X, Baker SC. Coronavirus endoribonuclease targets viral polyuridine sequences to evade activating host sensors. Proc Natl Acad Sci U S A. 2020; 117(14): 8094-8103.

10. Ortiz-Alcantara J, Bhardwaj K, Palaninathan S, et al. Small molecule inhibitors of the SARS-CoV Nsp15 endoribonuclease. Virus Adapt Treat. 2010; 2: 125-133.

11. Pillon MC, Frazier MN, Dillard LB, et al. Cryo-EM structures of the SARS-CoV-2 endoribonuclease Nsp15 reveal insight into nuclease specificity and dynamics. Nat Commun. 2021; 12: 636.

12. Kim Y, Wower J, Maltseva N, et al. Tipiracil binds to uridine site and inhibits Nsp15 endoribonuclease NendoU from SARS-CoV-2. Communications Biology. 2021; 4: 193. 
13. Sinha SK, Shakya A, Prasad SK, et al. An in-silico evaluation of different Saikosaponins for their potency against SARS-CoV-2 using NSP15 and fusion spike glycoprotein as targets. J Biomol Struct Dyn. 2020 May 13; 1-12.

14. Chandra A, Gurjar V, Qamar I, et al. Identification of potential inhibitors of SARS-COV2 endoribonuclease (EndoU) from FDA approved drugs: a drug repurposing approach to find therapeutics for COVID-19. J Biomol Struct Dyn. 2020; 1-11.

15. Jo S, Kim T, Iyer VG, Im W. CHARMM-GUI: a web-based graphical user interface for CHARMM. J Comput Chem. 2008;29(11):1859-65.

16. Brooks BR, Brooks CL 3rd, Mackerell AD Jr, et al. CHARMM: the biomolecular simulation program. J Comput Chem. 2009;30(10):1545-1614.

17. J. Lee, X. Cheng, J.M. Swails, M.S. Yeom, P.K. Eastman, J.A. Lemkul, S. Wei, J. Buckner, J.C. Jeong, Y. Qi, S. Jo, V.S. Pande, D.A. Case, C.L. Brooks III, A.D. MacKerell Jr, J.B. Klauda, and W. Im. CHARMM-GUI Input Generator for NAMD, GROMACS, AMBER, OpenMM, and CHARMM/OpenMM Simulations using the CHARMM36 Additive Force Field. Journal of Chemical Theory and Computation. 2016;12(1):405-413

18. Andersen PI, Ianevski A, Lysvand $\mathrm{H}$, et al. Discovery and development of safe-in-man broad-spectrum antiviral agents. Int J Infect Dis. 2020; 93: 268-276.

19. Petrowsky H, Roberts GD, Kooby DA, et al. Functional interaction between fluorodeoxyuridine-induced cellular alterations and replication of a ribonucleotide reductase-negative herpes simplex virus. J Virol. 2001; 75(15): 7050-7058.

20. Schor S, Einav S. Repurposing of kinase inhibitors as broad-spectrum antiviral drugs. DNA Cell Biol. 2018; 37(2): 63-69.

21. Kumar N, Sharma NR, Ly H, et al. Receptor tyrosine kinase inhibitors that block replication of influenza A and other viruses. Antimicrob Agents Chemother. 2011; 55(12): 5553-5559.

22. Hondermarck H, Bartlett NW, Nurcombe V. The role of growth factor receptors in viral infections: An opportunity for drug repurposing against emerging viral diseases such as COVID-19? FASEB BioAdv. 2020; 2(5): 296-303.

23. Dyall J, Coleman CM, Hart BJ, et al. Repurposing of clinically developed drugs for treatment of Middle East respiratory syndrome coronavirus infection. Antimicrob Agents Chemother. 2014; 58(8): 4885-4893. 
24. Abruzzese E, Luciano L, D’Agostino F, et al. SARS-CoV-2 (COVID-19) and Chronic Myeloid Leukemia (CML): a case report and review of ABL kinase involvement in viral infection. Mediterr J Hematol Infect Dis. 2020; 12(1): e2020031.

25. Raymonda, MH, Ciesla, JH, Monaghan $\mathrm{M}$, et al. Pharmacologic profiling reveals lapatinib as a novel antiviral against SARS-CoV-2 in vitro. BioRxiv. Preprint. https://doi.org/10.1101/2020.11.25.398859.

26. Shema Mugisha C, Vuong HR, Puray-Chavez M, et al. A simplified quantitative realtime PCR assay for monitoring SARS-CoV-2 growth in cell culture. mSphere. 2020; 5(5): e00658-20.

27. Jácome R, Campillo-Balderas JA, Ponce de León S, et al. Sofosbuvir as a potential alternative to treat the SARS-CoV-2 epidemic. Sci Rep. 2020; 10: 9294. 


\section{SUPPLEMENTAL FIGURES}

Table S1. List of random compounds sorted by their energies of interaction with SARS-CoV-2

NSP15 in the docked positions. DFE: Docking free energy.

\begin{tabular}{|l|l|l|l|}
\hline Drug Name & $\begin{array}{l}\text { DFE } \\
\text { kcal/mol }\end{array}$ & Drug Name & $\begin{array}{l}\text { DFE } \\
\text { kcal/mol }\end{array}$ \\
\hline Gadoxetate & -8.31 & Erythrityl tetranitrate & -5.97 \\
\hline Iohexol & -7.45 & Trihexyphenidyl & -5.89 \\
\hline Chlortetracycline & -7.11 & Aldehydo-N-Acetyl-D-Glucosamine & -5.82 \\
\hline Lurasidone & -6.99 & Physostigmine & -5.78 \\
\hline Bosutinib & -6.98 & Clotrimazole & -5.57 \\
\hline Phloxine B & -6.84 & Valproate bismuth & -5.48 \\
\hline Linagliptin & -6.76 & Mefloquine & -5.43 \\
\hline Permethrin & -6.72 & Boscalid & -5.37 \\
\hline Alfuzosin & -6.70 & Amoxapine & -5.32 \\
\hline Bazedoxifene & -6.55 & Chlorphenesin & -5.30 \\
\hline Pimozide & -6.53 & Dexbrompheniramine & -5.29 \\
\hline Colchicine & -6.51 & Resveratrol & -5.26 \\
\hline Benoxaprofen & -6.50 & Clonazepam & -5.24 \\
\hline Paliperidone & -6.49 & Pentolinium & -5.17 \\
\hline Lutein & -6.49 & Lamotrigine & -5.11 \\
\hline Isoxaflutole & -6.39 & Haloprogin & -5.08 \\
\hline Gemfibrozil & -6.38 & Bupropion & -4.99 \\
\hline Dexlansoprazole & -6.36 & Phendimetrazine & -4.94 \\
\hline Flecainide & -6.19 & L-Histidine & -4.92 \\
\hline Tetrabenazine & -6.19 & Apraclonidine & -4.88 \\
\hline Amisulpride & -6.18 & Pemoline & -4.78 \\
\hline Gentian Violet Cation & -6.15 & Propylhexedrine & -4.73 \\
\hline Meclofenamic acid & -6.15 & Clioquinol & -4.71 \\
\hline Perampanel & -6.12 & Eugenol & -4.67 \\
\hline Ribostamycin & -6.07 & Taurine & -4.48 \\
\hline Ospemifene & -6.00 & Sevoflurane & -4.32 \\
\hline Lacosamide & -5.97 & Urea & -3.55 \\
\hline Thioridazine & -5.97 & Ethyl chloride & -3.24 \\
\hline & & & \\
\hline
\end{tabular}

